\title{
Using emergency department-based inception cohorts to determine genetic characteristics associated with long term patient outcomes after motor vehicle collision: Methodology of the CRASH study
}

Timothy F Platts-Mills ${ }^{1,2^{*}}$, Lauren Ballina ${ }^{1}$, Andrey V Bortsov' ${ }^{1}$, April Soward' ${ }^{1}$, Robert A Swor ${ }^{3}$, Jeffrey S Jones ${ }^{4}$, David C Lee ${ }^{5}$, David A Peak ${ }^{6}$, Robert M Domeier ${ }^{7}$, Niels K Rathlev ${ }^{8}$, Phyllis L Hendry ${ }^{9}$ and Samuel A McLean ${ }^{1,2}$

\begin{abstract}
Background: Persistent musculoskeletal pain and psychological sequelae following minor motor vehicle collision (MVC) are common problems with a large economic cost. Prospective studies of pain following MVC have demonstrated that demographic characteristics, including female gender and low education level, and psychological characteristics, including high pre-collision anxiety, are independent predictors of persistent pain. These results have contributed to the psychological and social components of a biopsychosocial model of post-MVC pain pathogenesis, but the biological contributors to the model remain poorly defined. Recent experimental studies indicate that genetic variations in adrenergic system function influence the vulnerability to post-traumatic pain, but no studies have examined the contribution of genetic factors to existing predictive models of vulnerability to persistent pain.

Methods/Design: The Project CRASH study is a federally supported, multicenter, prospective study designed to determine whether variations in genes affecting synaptic catecholamine levels and alpha and beta adrenergic receptor function augment social and psychological factors in a predictive model of persistent musculoskeletal pain and posttraumatic stress disorder (PTSD) following minor MVC. The Project CRASH study will assess pain, pain interference and PTSD symptoms at 6 weeks, 6 months, and 1 year in approximately 1,000 patients enrolled from 8 Emergency Departments in four states with no-fault accident laws.

Discussion: The results from this study will provide insights into the pathophysiology of persistent pain and PTSD following MVC and may serve to improve the ability of clinicians and researchers to identify individuals at high risk for adverse outcomes following minor MVC.
\end{abstract}

\section{Background}

During recent decades, emergency medicine research has continued to expand in scope and increasingly focuses not only on immediate patient outcomes but also on patient outcomes well beyond emergency department (ED) departure. Inception cohort studies examining long term outcomes among patients experiencing acute illness

\footnotetext{
* Correspondence: tplattsm@med.unc.edu

'Department of Anesthesiology, University of North Carolina, Chapel Hill, North Carolina, USA

Full list of author information is available at the end of the article
}

or trauma benefit from enrolling patients in the ED because it is the site of their initial care. In addition, for many patients the ED serves as their only source of care $[1,2]$. For these reasons, an increasing number of large scale multidisciplinary research projects have utilized ED-based inception cohorts to evaluate long term outcomes after screening, risk stratification, or interventions performed in the ED [3-8].

This report describes the methods of Project CRASH, an example of a type of ED-based inception cohort study which we anticipate will become increasingly 
common in the future. Project CRASH uses an EDbased inception cohort to enroll patients soon after injury, and examines genetic factors associated with long term patient outcomes. A prior study examined genetic variations in alcohol metabolism as a risk factor for trauma center admission [9], but to our knowledge no studies have used ED-based inception cohorts to examine genetic characteristics associated with long term patient outcomes in the hope of advancing understanding of disease pathophysiology.

The goal of Project CRASH is to gain new insights into the pathophysiology of persistent pain and psychological sequelae after motor vehicle collision (MVC). Approximately 6 million people present to United States EDs each year for care after MVC. More than $90 \%$ of these individuals do not have serious physical injury and are discharged to home after ED evaluation [10]. However, persistent post-MVC pain (most commonly neck pain) is experienced by $10-20 \%$ of individuals after "minor" MVC $[11,12]$ with significant associated economic cost [13]. The mechanisms by which patients develop acute and chronic pain after minor MVC remain poorly understood [14]. Interestingly, increasing evidence suggests that stress systems, such as the sympathetic nervous system and adrenomedullary hormonal system, may modulate neurosensory processing after trauma exposure, contributing to the development of chronic pain [15]. If a particular physiologic system is involved in the pathogenesis of a disorder, then genetic variations influencing the function of this system would be expected to be associated with vulnerability to develop the disorder and to improve prediction of which individuals will develop this disorder. These concepts are the rationale of the Project CRASH, R01AR056328, "Genetic Predictors of Acute and Chronic Musculoskeletal Pain after Motor Vehicle Collision."

\section{Methods/Design Study Sites}

The CRASH study is a prospective multicenter observational cohort study of patients experiencing minor MVC. Study participants are enrolled at research network ED sites and receive initial interview evaluation at the time of the ED visit. Study participant follow-up assessments are performed at 6 weeks, 6 months, and one year. The study research network ("TRYUMPH Research Network") includes Baystate Medical Center, Massachusetts General Hospital, North Shore University Hospital, Shands Jacksonville Hospital, Spectrum Health Butterworth Hospital, St. Joseph Mercy Hospital, and William Beaumont Hospital (2 ED sites). All eight EDs are located in states with "no fault" insurance laws that restrict the right to seek recovery from other parties through the civil-justice system. The study is restricted to states with no-fault accident laws to minimize the likelihood of legal action following MVC, which is a potential confounding factor influencing patient outcome [16]. The data coordinating center for the study is located at the University of North Carolina, Chapel Hill, NC, USA.

\section{Inclusion Criteria}

Patients age 18 to 65 who present to the ED within 24 hours after minor MVC and who are unlikely to require admission are screened for eligibility. Patients with injuries likely to require hospitalization, fractures (other than small bone fractures), major lacerations (defined as a laceration more than $20 \mathrm{~cm}$ in length or more than four lacerations requiring sutures), intracranial injury, or spinal injury (defined as vertebral fracture or dislocation, or new neurologic deficit) are excluded. Patients who are deemed eligible but subsequently admitted overnight to the hospital are excluded. Patients who go to an ED observation area for a brief period (e.g. "6 hour rule out") remain eligible. Patients are excluded if they are prisoners, pregnant, not alert and oriented, or unable to read and understand English. Patients are also excluded if they take $\beta$-receptor antagonist or if they take opioids on a daily basis above a total daily dose of $20 \mathrm{mg}$ of oxycodone or equivalent. In addition, due to the effects of ethnicity on genetic risk factor assessment (population stratification bias [17] that may require different ethnicities to be analyzed separately) and budget restrictions limiting sample size, enrollment is limited to European Americans. After assessment for eligibility, signed informed consent is obtained from all patients enrolled in the study.

\section{Patient screening and consent}

Patient screening and assessment are performed using web-based study assessment forms. Research assistants (RAs) complete a web-based patient screening questionnaire for each patient presenting to the ED for evaluation after MVC during day and evening hours when study site research team members are staffing the ED. The screening form prompts the RA to complete a series of questions. If participants are eligible for participation based on screening questionnaire responses, the RA is automatically advanced to the ED assessment interview web survey. If participants are not eligible, the reason for ineligibility is stored by the system. If patients are eligible, they are offered participation in the study. Signed informed consent is obtained from willing participants.

\section{Blood collection for DNA}

After consent is obtained, a single blood sample $(8.5 \mathrm{cc})$ is collected using a PAXgene DNA storage tube (http:// www.preanalytix.com). When possible, this blood sample is obtained when blood is collected as part of the 
patient's medical evaluation, to avoid additional phlebotomy. Each blood specimen is labeled with a barcode sticker, which serves as a unique identifier for the sample. After the barcode sticker is placed on the sample, the barcode is scanned using a reader wand which enters the barcode number into a web-based tracking system and links the number with the participant's identification number. The barcode is also scanned at the time of shipment from the study site to the genotyping facility, and at the time of receipt by the genotyping facility, to maintain blood sample chain of custody. PAXgene DNA storage tubes are stored at $4^{\circ} \mathrm{C}$ (standard refrigeration) for up to two weeks [18] at the study site prior to batch shipment to Cogenics, Inc., (Morrisville, $\mathrm{NC}$ ).

\section{ED Interview}

ED assessments are conducted by trained research assistants using a standardized web-based questionnaire on laptop computer. Back-up paper copies are used by RAs if hospital wireless internet service is unavailable. The ED interview begins with the collection of patient contact information, including information on two potential alternative contacts. Subsequent interview assessments include the collection of detailed information regarding the collision event, current somatic and psychological symptoms, past somatic and psychological symptoms, and general health and medication use (Table 1; Additional Files 1 and 2). Participants are compensated $\$ 80$ for completing the ED evaluation.

\section{Data Extraction}

During the week following the completion of the ED interview, study site RAs extract data from the participant's medical record using a standardized web-based data extraction form. Fields on this data extraction form provide explicit definitions of all variables. Information collected includes chief complaint, vital signs, past medical history, medications given in the ED and prescribed at discharge, injuries described in the medical record, and results of radiologic studies performed in the ED. All aspect of the emergency medical record including physician notes, nursing notes, and physician orders were used for completion of data extraction. Injury information was used to generate Abbreviated Injury Severity Scores [19].

\section{Follow-Up Assessments}

Follow-up assessments are completed at 6 weeks, 6 months, and 1 year post-enrollment with assessment made during a 4 week window for each follow-up time point (Additional File 3). Information collected at each follow-up is identical, except that drug and alcohol use is reassessed at only the 6 month and 1 year time points. Participants are able to complete the interview online, by telephone, or by mail. Information packets are sent that remind participants that it is time to complete their next assessment. These packets include the actual questionnaires to be completed during the interview; if participants choose to complete their assessment via mail they can do so or they can follow along with the questionnaires at home during a telephone interview. Follow up interviews are scheduled at a time (day or evening) that is convenient for the participant. Three and nine months after enrollment, participants also complete a contact information update via mail. Assessments collect detailed information regarding re-injury or new injury events, as well as information regarding involvement in a litigation or disability claim, missed work or other activities, somatic symptoms (including pain symptoms), psychological symptoms, and functional interference due to pain (Table 1). For any body region for which pain is reported, the patient is also asked whether the pain is due to (began after) the MVC. Health care utilization assessments are also evaluated and include: medication use; visits to the ED, primary physician, specialist physicians, or alternative medicine providers; and hospitalization. Participants are compensated $\$ 50, \$ 60$, and $\$ 70$ after completing the 6 week, 6 month, and 1 year follow-up interviews, respectively.

\section{Protection of Patient Privacy}

A number of methods are used to maintain the confidentiality of study data. A Certificate of Confidentiality has been obtained from the National Institutes of Health to protect identifiable research information from forced disclosure [20]. All research assistants involved in the study complete training in the protection of patient confidentiality through the Collaborative Institutional Training Initiative [21]. Signed consent is obtained from all patients to perform all assessments and to allow future analysis of de-identified blood specimens. A unique patient identifier is used to link patient identifying information to results of questionnaires and genetic information, and all analyses are conducted on de-identified data. The study has been approved by the institutional review boards of all participating institutions.

\section{Sample Size Estimate}

Sample size was calculated to determine the number of patients needed to assess the influence of selected adrenergic system-related genes on the presence of persistent moderate or severe neck pain with adequate power. Because the genes to be assessed contain approximately 59 haplotypes, a Bonferroni corrected alpha was set at. $00085(.05 / 59)$. Estimates of the number of patients needed to identify the effect sizes obtained for candidate haplotypes examined in our pilot data ranged from 171 
Table 1 Study question domains, specific measures, and times of assessment.

\begin{tabular}{|c|c|c|c|c|c|}
\hline Domain & Measure & ED & $1 \mathrm{M}$ & $6 \mathrm{M}$ & $1 Y^{*}$ \\
\hline MVC injury events & Questionnaire & $\bullet$ & & & \\
\hline \multirow[t]{2}{*}{ Current pain symptoms } & NRS**, Regional Pain Scale & $\bullet$ & $\bullet$ & $\bullet$ & $\bullet$ \\
\hline & Scale[25] & & & & \\
\hline Optimism & Life Orientation Test-Revised[26] & $\bullet$ & & & \\
\hline Current whiplash symptoms & Quebec classification[1 1] & $\bullet$ & & & \\
\hline Current somatic symptoms & Standardized questionnaire & $\bullet$ & $\bullet$ & $\bullet$ & $\bullet$ \\
\hline Distress after MVC & Peritraumatic Distress Inventory[27] & $\bullet$ & & & \\
\hline Dissociative symptoms after MVC & Critical Events Perception Inventory[28] & $\bullet$ & & & \\
\hline Catastrophizing & Pain Catastrophizing scale[29] & $\bullet$ & & & \\
\hline Current somatic symptoms & Somatic symptom interview[30] & & & & \\
\hline Pre-MVC General health & SF-12[31] & $\bullet$ & & & \\
\hline Pre-MVC Anxiety symptoms & STPI ${ }^{*}$ Form Y[32] & $\bullet$ & & & \\
\hline Pre-MVC Depressive symptoms & CES-Dํ3] & $\bullet$ & & & \\
\hline $\mathrm{PTSD}^{\dagger}$ symptoms & Impact of Event Scale-Revised[34] & & $\bullet$ & $\bullet$ & $\bullet$ \\
\hline General health & SF-12[31] & & $\bullet$ & $\bullet$ & $\bullet$ \\
\hline Demographic information & Standard items & $\bullet$ & & $\bullet$ & $\bullet$ \\
\hline Medication Use & Standard items & $\bullet$ & $\bullet$ & $\bullet$ & $\bullet$ \\
\hline Alcohol and drug use & TWEAK[35], Substance Abuse Outcomes Module[36] & $\bullet$ & & $\bullet$ & $\bullet$ \\
\hline New injury or re-injury & Standard Items & & $\bullet$ & $\bullet$ & $\bullet$ \\
\hline Pain interference & Brief Pain Inventory (pain interference questions)[37] & & $\bullet$ & $\bullet$ & $\bullet$ \\
\hline Service utilization, disability/litigation claims & Standard items & & $\bullet$ & $\bullet$ & $\bullet$ \\
\hline
\end{tabular}

${ }^{*} \mathrm{ED}=$ Emergency Department, $1 \mathrm{M}=1$ month, $6 \mathrm{M}=6$ months, $1 \mathrm{Y}=\mathrm{w}$ year, ${ }^{* *}$ NRS $=$ Numeric rating scale, ${ }^{*}$ State Trait Personality Inventory, ${ }^{\dagger} \mathrm{PTSD}=$

Posttraumatic Stress Disorder, ${ }^{\circ}$ Center for Epidemiologic Studies Depression Scale

to 1,295 (alpha $=.00085$, beta $=.8$ ). These estimates were believed to be conservative because 1) the inclusion of non-genetic factors should reduce the unexplained variance in the model and increase power to detect the influence of genetic factors, 2) analyses will utilize repeated-measures logistic regression, which will increase power, and 3) for some genes, only specific risk haplotypes will be assessed so the actual number of haplotypes will likely be less than 59 . Based on these analyses, recruitment of 936 patients is planned, in order to achieve at least 795 patients completing follow-up time points (estimated follow-up rate of $85 \%$ ).

\section{Data Analysis}

DNA is extracted (average PAXgene DNA yield $150 \mu \mathrm{g}$ to $500 \mu \mathrm{g}$ ) and targeted genotyping of single nucleotide polymorphisms (SNPs) is performed using the Sequenom (Sequenom, Inc., San Diego, CA) platform. When possible, a haplotype-based approach to genetic analyses is utilized, because previous studies suggest that a haplotype-based approach to genetic analyses is often most useful. This is because the overall functional state of a gene may not be easily deduced from information regarding a single SNP [22]. For example, haplotypedependent secondary RNA structure can have a much greater influence on function than a functional SNP within this haplotype [22]. To construct haplotypes, both functional SNPs previously shown to affect gene function and also tag SNP markers within each gene locus (to capture haplotypic diversity) are genotyped. Two hapmap samples, for which the entire genome sequence is known, and 2 repeat samples are included in each genotyping batch to assess genotypic accuracy and reliability. Haplotypes are then constructed for each of the genetic risk factors assessed using the Haploview software program. Polymorphisms of interest include genetic variations influencing catecholamine levels (monoamine oxidase A, monoamine oxidase B, norepinephrine transporter, catechol-O-methyltransferase) and adrenoreceptor function $(\alpha 1 \mathrm{~A}, \alpha 1 \mathrm{~B}, \alpha 1 \mathrm{D}, \alpha 2 \mathrm{~A}, \alpha 2 \mathrm{~B}$, $\alpha 2 C, \beta 1, \beta 1)$. Quality control of genetic data includes assessment of call rates for each SNP, identification of samples with call rates $<90 \%$, and test of Hardy-Weinberg equilibriums for each locus. Genetic data results are then merged with phenotypic data and analyzed using standard statistical methods. Primary and secondary analyses evaluate genotypic and phenotypic predictors of persistent pain and psychological sequelae.

\section{Discussion}

ED-based inception cohort studies such as Project $\mathrm{CRASH}$, which combine genotypic and phenotypic data collection, are likely to become increasingly common for several reasons. First, as the cost of genotyping 
continues to decline, and as our knowledge of genetic polymorphisms influencing physiologic processes continues to increase, the value (cost effectiveness) of these studies will continue to grow. Second, genetic (and epigenetic) techniques represent one useful method to address a critical shortfall in current medical knowledge: our ability to identify those at high risk for a particular disease outcome has rapidly outpaced our knowledge of the mechanisms mediating the outcome. That is, increasingly we can identify "who", but we do not know "why". Including genetic factors in traditional epidemiologic studies enhances the ability of study findings to yield clues regarding disease pathogenesis, by determining the physiologic systems or pathways contributing to disease outcomes, and by determining the environmental conditions under which these outcomes occur (gene $x$ environment interactions). Further knowledge of the physiologic systems which mediate disease outcomes has the potential to enhance our ability to identify high-risk individuals and/or to suggest novel preventive interventions. These studies require relatively large number of patients, which ED-based networks can provide.

To most rapidly bring the benefits of these types of studies to our patients, organizations within academic emergency medicine should continue to look for opportunities to bring knowledge of genetic epidemiologic methods to emergency medicine researchers. The above description of Project CRASH is hopefully useful in this regard. Fortunately, the genetics field continues to grow exponentially, with an increasing array of opportunities to rapidly get "up to speed" regarding performing and interpreting genetics studies. For example, there are now a number of societies focused on genetic studies in humans (e.g. Society for Human Genetics), and online genetic education resources are available through the National Human Genome Research Institute (http:// www.genome.gov/10000464), the Human Genome Project (http://www.ornl.gov/sci/techresources/Human_Genome/posters/chromosome/), and other educational organizations.

\section{Difficulties and Potential Limitations}

A current limitation of epidemiologic studies examining genetic factors is that they often must be performed within a particular ethnicity. As described above, Project CRASH is limited to European Americans only. This is because the prevalence of specific genetic variations often varies between ethnicities. For example, haplotype frequencies for COMT genes are different between Caucasians, African Americans, and Asians [23]. This necessitates stratified analyses, and the size of each strata must be large, often limiting a study to a single strata within the context of the budget of a particular grant mechanism.
Several methods are now available to address this problem of population stratification. One of them is principal components analysis of a set of ancestry-informative markers (i.e. SNPs which have different frequencies among populations from different regions) to identify population ancestral heterogeneity [24]. However, the accuracy of these methods vs. stratified analyses remains a matter of debate. As with other areas of genetic research, the only constant is that techniques and methods will continue to rapidly change.

\section{Additional material}

Additional file 1: CRASH Methods ED Assessment Part 1. Part 1 of the 2 part survey administered in the Emergency Department.

Additional file 2: CRASH Methods ED Assessment Part 2. Part 2 of the 2 part survey administered in the Emergency Department.

Additional file 3: CRASH Methods Follow-Up Questionnaire. The survey administered at each follow-up interval.

\section{Acknowledgements}

Funding for this study was provided by NIH 5R01AR056328-02. Dr. PlattsMills' time is covered in part by NIH 5KL2 RR025746-03.

\section{Author details}

'Department of Anesthesiology, University of North Carolina, Chapel Hill, North Carolina, USA. ${ }^{2}$ Department of Emergency Medicine, University of North Carolina, Chapel Hill, North Carolina, USA. ${ }^{3}$ Department of Emergency Medicine, William Beaumont Hospital, Royal Oak, Michigan, USA.

${ }^{4}$ Department of Emergency Medicine, Spectrum Health - Butterworth Campus, Grand Rapids, Michigan, USA. ${ }^{5}$ Department of Emergency Medicine, North Shore University Hospital, Manhasset, New York, USA. ${ }^{6}$ Department of Emergency Medicine, Massachusetts General Hospital, Boston, Massachusetts, USA. ${ }^{7}$ Department of Emergency Medicine, St. Joseph Mercy Hospital, Ann Arbor, Michigan, USA. ${ }^{8}$ Department of Emergency Medicine, Baystate Medical Center, Springfield, Massachusetts, USA. ${ }^{9}$ Department of Emergency Medicine and Pediatrics, University of Florida-Jacksonville, Jacksonville, Florida, USA.

\section{Authors' contributions}

SM developed the study concept and design. LB, AS, RS, JJ, DL, DP, RD, NR, and $\mathrm{PH}$ contributed to the acquisition of data and supervised the study. $A B$ provided statistical expertise and assisted in study design. TPM and SM drafted the manuscript, and all authors made critical revisions of the manuscript for important intellectual content. TPM and SM take responsibility for the paper as a whole. All authors read and approved the final manuscript.

\section{Competing interests}

The authors declare that they have no competing interests.

Received: 10 April 2011 Accepted: 26 September 2011 Published: 26 September 2011

\section{References}

1. Tang N, Stein J, Hsia RY, Maselli JH, Gonzales R: Trends and characteristics of US emergency department visits, 1997-2007. JAMA 304(6):664-670.

2. Medicaid beneficiaries and access to care. Kaiser Commission on Medicaid and the Uninsured, Kaiser Family Foundation. [http://www.kff. org/medicaid/upload/8000-02.pdf].

3. Maio RF, Shope JT, Blow FC, Gregor MA, Zakrajsek JS, Weber JE, Nypaver MM: A Randomized Controlled Trial of an Emergency Department-Based Interactive Computer Program to Prevent Alcohol 
Misuse Among Injured Adolescents. Annals of Emergency Medicine 2005, 45(4):420-429

4. Walton MA, Chermack ST, Shope JT, Bingham CR, Zimmerman MA, Blow FC, Cunningham RM: Effects of a Brief Intervention for Reducing Violence and Alcohol Misuse Among Adolescents. Journal of the American Medical Association 2010, 304(5):527-535.

5. Kline JA, Courtney DM, Kabrhel C, Moore CL, Smithline HA, Plewa MC, Richman PB, O'Neil BJ, Nordenholz K: Prospective multicenter evaluation of the pulmonary embolism rule-out criteria. J Thromb Haemost 2008, 6(5):772-780.

6. Courtney DM, Kline JA, Kabrhel C, Moore CL, Smithline HA, Nordenholz KE, Richman PB, Plewa MC: Clinical features from the history and physical examination that predict the presence or absence of pulmonary embolism in symptomatic emergency department patients: results of a prospective, multicenter study. Annals of Emergency Medicine 2010, 56(5).

7. Haukoos JS, Hopkins E, Conroy AA, Silverman M, Byyny RL, Eisert S, Thrun MW, Wilson ML, Hutchinson AB, Forsyth J, et al: Routine opt-out rapid HIV screening and detection of HIV infection in emergency department patients. Journal of the American Medical Association 2010, 304(3):284-292.

8. Trautman DE, McCarthy ML, Miller N, Campbell JC, Kelen GD: Intimate partner violence and emergency department screening: computerized screening versus usual care. Annals of Emergency Medicine 2007, 49(4):526-534.

9. Tseng YM, Hu BW, Tsai SM, I.J C, Jin YR, Lee JH, Huang FD, Wu SH, Yeh FL, Ysai LY: Distribution of alcohol-metabolizing enzyme genotypes in trauma patients with excessive alcohol consumption in the emergency department. Clin Biochem 2007, 40(5-6):370-376.

10. McCaig LF: National Center for Health Statistics. 2010.

11. Hartling L, Brison RJ, Ardern C, Pickett W: Prognostic value of the Quebec Classification of Whiplash-Associated Disorders. Spine 2001, 26(1):36-41.

12. Suissa $S$, Harder $S$, Veilleux M: The relation between initial symptoms and signs and the prognosis of whiplash. European Spine Journal 2001, 10(1):44-49.

13. Freeman MD, Croft AC, Rossignol AM, Weaver DS, Reiser M: A review and methodologic critique of the literature refuting whiplash syndrome. Spine 1999, 24(1):86-96.

14. Carroll L, Holm LW, Hogg-Johnson S, Cote P, Cassidy JD, Haldeman S, Nordin M, Hurwitz EL, Carragee EJ, van der Velde G, et al: Course and prognostic factors for neck pain in whiplash-associated disorders (WAD): results of the Bone and Joint Decade 2000-2010 Task Force on Neck Pain and Its Associated Disorders. Spine (Phila Pa 1976) 2008, 33(4 Suppl): S83-92.

15. McLean SA, Clauw DJ, Abelson JL, Liberzon I: The development of persistent pain and psychological morbidity after motor vehicle collision: integrating the potential role of stress response systems into a biopsychosocial model. Psychosom Med 2005, 67(5):783-790.

16. Cassidy JD, Carroll LJ, Cote P, Lemstra M, Berglund A, Nygren A: Effect of eliminating compensation for pain and suffering on the outcome of insurance claims for whiplash injury. New England Journal of Medicine 2000, 342(16):1179-1186.

17. Diatchenko L, Nackley AG, Slade GD, Belfer I, Max MB, Goldman D, Maixner W: Responses to Drs. Kim and Dionne regarding comments on Diatchenko, et al. Catechol-O-methyltransferase gene polymorphisms are associated with multiple pain-evoking stimuli. Pain 2007, 129(3):366-370.

18. PAXgene Blood DNA Kit Handbook. PreAnalytix Company 2009.

19. The Abbreviated Injury Scale (AIS) 1990 Revision. Des Plaines, IL: Association for the Advancement of Automotive Medicine; 1990.

20. Certificate of Confidentiality. [http://grants.nih.gov/grants/policy/coc/].

21. CITI Program. [https://www.citiprogram.org/default.asp?language=english].

22. Nackley AG, Shabalina SA, Tchivileva IE, Satterfield K, Korchynskyi O, Makarov SS, Maixner W, Diatchenko L: Human catechol-Omethyltransferase haplotypes modulate protein expression by altering mRNA secondary structure. Science 2006, 314(5807):1930-1933.

23. DeMille MM, Kidd JR, Ruggeri V, Palmatier MA, Goldman D, Odunsi A, Okonofua F, Grigorenko E, Schulz LO, Bonne-Tamir B, et al: Population variation in linkage disequilibrium across the COMT gene considering promoter region and coding region variation. Hum Genet 2002, 111(6):521-537.
24. Price AL, Patterson NJ, Plenge RM, Weinblatt ME, Shadick NA, Reich D: Principal components analysis corrects for stratification in genome-wide association studies. Nat Genet 2006, 38(8):904-909.

25. Wolfe F: Pain extent and diagnosis: development and validation of the regional pain scale in 12,799 patients with rheumatic disease. Rheumatol 2003, 30(2):369-378.

26. Hirsch JK, Britton PC, Conner KR: Psychometric evaluation of the Life Orientation Test-Revised in treated opiate dependent individuals. International Journal of Mental Health and Addiction 2010, 8(3):423-431.

27. Brunet A, Weiss DS, Metzler TJ, Best SR, Neylan TC, Rogers C, Fagan J, Marmar CR: The Peritraumatic Distress Inventory: a proposed measure of PTSD criterion A2. American Journal of Psychiatry 2001, 158(9):1480-1485.

28. Michaels AJ, Michaels CE, Moon CH, Smith JS, Zimmerman MA, Taheri PA, Peterson C: Posttraumatic stress disorder after injury: impact on general health outcome and early risk assessment. Journal of Trauma-Injury Infection \& Critical Care 1999, 47(3):460-466, discussion 466-467.

29. Sullivan MJ, Bishop S, Pivik J: The Pain Catastrophizing Scale: development and validation. Psychological Assessment 1995, 7:524-532.

30. Pennebaker JW: The Psychology of Physical Symptoms. New York: Springer-Verlag; 1982.

31. Ware J Jr, Kosinski M, Keller SD: SF-12: A 12-item Short-Form Health Survey: Construction of scales and preliminary tests of reliability and validity. Medical Care 1996, 34:220-233.

32. Spielberger CD: Preliminary manual for the State - Trait Personality Inventory (STPI). 2000

33. Radloff $L S$ : The CES-D Scale: A self-report depression scale for research in the general population. Applied Psychological Measurement 1977, 1:385-401.

34. Weiss D: Psychometric review of the Impact of Event Scale-Revised. Lutherville, Maryland: Sidran Press; 1996.

35. Cherpitel CJ: Screening for alcohol problems in the emergency room: a rapid alcohol problems screen. Drug \& Alcohol Dependence 1995, 40(2):133-137.

36. Smith GRJ, Ross RL, KM R: Psychiatric outcomes module: Substance abuse outcomes module (SAOM). In Outcomes assessment in clinical practice. Edited by: Sederer LI, Dickey B. Baltimore, MD: Williams 1996:85-88.

37. Keller S, Bann CM, Dodd SL, Schein J, Mendoza TR, Cleeland CS: Validity of the brief pain inventory for use in documenting the outcomes of patients with noncancer pain. Clin J Pain 2004, 20(5):309-318.

Pre-publication history

The pre-publication history for this paper can be accessed here: http://www.biomedcentral.com/1471-227X/11/14/prepub

doi:10.1186/1471-227X-11-14

Cite this article as: Platts-Mills et al: Using emergency departmentbased inception cohorts to determine genetic characteristics associated with long term patient outcomes after motor vehicle collision: Methodology of the CRASH study . BMC Emergency Medicine 2011 11:14

\section{Submit your next manuscript to BioMed Central and take full advantage of:}

- Convenient online submission

- Thorough peer review

- No space constraints or color figure charges

- Immediate publication on acceptance

- Inclusion in PubMed, CAS, Scopus and Google Scholar

- Research which is freely available for redistribution

Submit your manuscript at www.biomedcentral.com/submit
C Biomed Central 